\title{
On the Development and Testing of a Guided Ultrasonic Wave Array for Structural Integrity Monitoring
}

\author{
Paul Fromme, Paul D. Wilcox, Michael J. S. Lowe, and Peter Cawley, Member, IEEE
}

\begin{abstract}
The prototype of a guided ultrasonic wave array for the structural integrity monitoring of large, platelike structures has been designed, built, and tested. The development of suitably small transducers for the excitation and measurement of the first antisymmetric Lamb wave mode $A_{0}$ is described. The array design consists of a ring of 32 transducers, permanently bonded to the structure with a protective membrane, in a compact housing with the necessary multiplexing electronics. Using a phased addition algorithm with dispersion compensation and deconvolution in the wavenumber domain, a good dynamic range can be achieved with a limited number of transducers. Limitations in the transducer design and manufacture restricted the overall dynamic range achieved to $27 \mathrm{~dB}$. Laboratory measurements for a steel plate containing various defects have been performed. The results for standard defects are compared to theoretical predictions and the sensitivity of the array device for defect detection has been established. Simulated corrosion pitting and a defect cut with an angle grinder simulating general corrosion were detected.
\end{abstract}

\section{INTRODUCTION}

$\mathrm{M}$ ANY structures (e.g., off-shore oil platforms or oil storage tanks) are subject to adverse weather conditions, and thus should be inspected regularly for corrosion or the development of fatigue cracks during their service life. For the monitoring of the structural integrity of such large, remote, and difficult-to-access structures, it would be advantageous to permanently attach monitoring devices that can run autonomously (i.e., independent of external energy supply) and transmit data about the condition of the structure wirelessly [1]. An advantage of the permanent attachment of the device to the structure would be the possibility of comparative measurements. Taking measurements at different stages in the lifecycle of the structure, an emerging defect can be detected more clearly by comparison to an initial, defect-free measurement, thus increasing the probability of detection.

Guided ultrasonic waves, with stress distributed through the thickness of the material, can propagate along the thin and large plate-like components of such struc-

Manuscript received July 25, 2005; accepted November 18, 2005.

P. Fromme is with the Department of Mechanical Engineering, University College London, WC1E 7JE, UK (e-mail: p.fromme@ucl.ac.uk).

P. D. Wilcox is with the Department of Mechanical Engineering, University of Bristol, BS8 1TR, UK.

M. J. S. Lowe and P. Cawley are with the Department of Mechanical Engineering, Imperial College London, SW7 2AZ, UK. tures, and offer the potential to achieve the required permanent structural integrity monitoring [2] At a structural defect (e.g., severe thickness reduction due to corrosion pitting) the guided wave mode is scattered, and part of its energy is reflected back toward the monitoring location. This methodology has been successfully used for pipelines, which can be thought of as one-dimensional structures along which the energy of the guided ultrasonic wave propagates. Propagation distances of up to $100 \mathrm{~m}$ have been achieved, and testing equipment has been developed for efficient, nondestructive testing of pipelines and rails [3]. One of the main obstacles for one-dimensional structures has proven to be the controlled excitation and reception of selected guided wave modes, as even at low frequency, multiple modes can be present [4].

In thin plates and shells, the guided ultrasonic waves can propagate in two dimensions along the structure. Therefore, not only is it necessary to achieve a distinction between the different Lamb wave modes, but the twodimensional spreading of the guided ultrasonic wave needs to be taken into account. The angular resolution of the monitoring device has to be sufficient to distinguish between features in different directions on the structure. Furthermore, even without material damping, the amplitude of the guided wave decreases with distance from its source, as the energy spreads over a larger area. This results in the need for a sufficiently large dynamic range for the detection of small defects further away from the monitoring location than other prominent structural features. In order to avoid the double complication of angular resolution and multiple wave modes, most nondestructive testing applications work in the low-frequency thickness regime below the cut-off frequencies of the higher wave modes, in which only three guided wave modes $\left(\mathrm{S}_{0}, \mathrm{~A}_{0}, \mathrm{SH}_{0}\right)$ can exist [5]. Significant work has been reported on the first symmetrical mode $\mathrm{S}_{0}$ and the lowest shear mode $\mathrm{SH}_{0}$, as their displacement, stress distribution, and propagation velocity at low frequencies are similar to the bulk ultrasonic wave modes [6]. In particular, the $\mathrm{S}_{0}$ mode has been used for tomography applications [7], as at low frequency-thickness it has the highest group velocity, i.e., the fastest arrival time. The scattering at typical structural features and defects has been studied for a variety of applications [8], [9]. Recently, the development of a localized array based on the excitation and reception of the $\mathrm{S}_{0}$ mode using electromagnetic acoustical transducers (EMATs) acting as point sources/receivers has been reported [10]. 
This paper describes the development of a compact, low power array for permanent monitoring measurements based on the first antisymmetric, guided ultrasonic wave mode $\mathrm{A}_{0}$, using a similar data processing concept as [10]. The $\mathrm{A}_{0}$ mode at low frequencies has a significant outof-plane displacement component, and thus can be excited and received using simple piezoelectric transducer elements acting as point sources and receivers. The piezoelectric transducer elements have significantly smaller energy consumption than the EMATs used in [10], and they can be operated using battery power. This makes this measurement concept suitable for the long-term aim of the integration in a self-contained, wireless, battery-operated structural integrity monitoring scheme as outlined in [1].

A key point of this project has proven to be the development of suitably small $A_{0}$ mode transducers and their reliable and repeatable bonding with a protective membrane to the structure under investigation, a subject on which only limited work has been published [11]. The array concept and data processing follows largely the work by Wilcox et al. [10] and is only described conceptually in this paper. In order to limit the number of transducer elements and costs, combined transmit/receive transducer elements were used. The development and integration of the array transducer elements and electronics is described, and the practically achieved dynamic range compared to optimum theoretical predictions based on the array layout.

The feasibility of the measurement concept has been demonstrated in laboratory experiments using the developed, permanently attached array device on a steel plate, in which a variety of defects were machined as calibration defects and to simulate corrosion damage. The achieved sensitivity for detection of the various defects is compared to theoretical predictions taking the wave propagation, geometrical spreading, and scattering at the calibration defects into account. Simulated pits and irregular corrosion defects also were tested.

\section{Transducer Development}

\section{A. Transducer Design Requirements}

Building on a previous study [12], it was decided to use the $A_{0}$ mode below the cut-off frequencies of the higher order Lamb wave modes. This mode has a significant outof-plane displacement component and can be excited efficiently by piezoelectric disc transducer elements polarized in the thickness direction, applying a vertical force to the plate surface. This results in a propagating, flexural (bending) wave. For the autonomous operation of the device, running on battery power, these excitation transducers can be run with low power consumption. Due to good results in previous studies [13], it was decided to continue to work with the PZT material (Pz27, Ferroperm Piezoceramics A/S, Kvistgaard, Denmark) for the transducers.

The data processing concept detailed in the next section necessitates transducer elements acting as point sources and receivers to allow an omni-directional inspection of the structure. To achieve the same sensitivity and probability of detection in all directions from the array location, a circular array layout was used. For guided wave arrays, similar rules regarding the element spacing and overall size to avoid grating and side lobes exist [14] as for bulk ultrasonic wave arrays [15]. Therefore, the spacing between the transducer elements should not exceed about a third to half of the wavelength of the desired Lamb wave mode to avoid grating lobes. This limits the maximum diameter of the transducer elements to about a third of the wavelength of the guided wave. As the wavelength decreases with increasing excitation frequency, this effectively limits the maximum frequency that can be used with a chosen transducer element size.

Commonly, a higher inspection frequency (shorter wavelength) corresponds to a higher sensitivity for small defects. In order to avoid the complication of multiple wave modes, the maximum excitation frequency is often chosen well below the lowest cut-off frequency of the higher wave modes [16], in which the relative out-of-plane component of the $\mathrm{S}_{0}$ mode is also rather small. For the 5 -mm thick steel plate used in the laboratory experiments (Section IV), this limits the maximum frequency range to about $250 \mathrm{kHz}$, and gives a minimum wavelength in the order of $10 \mathrm{~mm}$. The design point of the array and transducer layout was chosen to lie in the frequency range of $100 \mathrm{kHz}$ to $240 \mathrm{kHz}$.

Results obtained from a preliminary array design using separate transducers for the excitation and reception of the guided waves [17] indicated a problem of coherent noise due to the large number of transducers. Each transducer bonded permanently to the structure acts as a small additional mass, at which a small part of the propagating guided wave is scattered. Thus it was decided to use combined transmit/receive transducer elements, reducing the overall number of elements to improve the dynamic range of the array layout. This increases the demands on the design of the transducers and the multiplexing electronics, but fewer transducer elements need to be manufactured.

In previous studies, individual piezoelectric discs were directly bonded to the structure using a thin film of flexible, two-component epoxy adhesive. Working well below the first resonance frequency, good and repeatable results were obtained in a reasonably wide frequency range with uniform transfer function [16]. For the purposes of this study, it was deemed necessary to include a membrane to protect the transducers against environmental influences in a field-deployment situation. This also had the benefit of reducing the extent of $\mathrm{S}_{0}$ mode generation and improved reliability of electrical connections and element positioning as discussed below. Another requirement was to construct the array in such a way that the assembled array could be attached to the structure as a unit with limited surface preparation, avoiding time-consuming and complicated assembly work in the field under unpredictable weather conditions. Due to these operational and deployment requirements, several options for the transducer design were explored, including various bonding and membrane materi- 
als, and the influence of backing masses and applied force to increase the efficiency and repeatability of the transducer elements.

\section{B. Coupling Membrane and Bonding}

The coupling membrane needs to fulfill three purposes: protect the transducer elements against environmental influences, accommodate uneven surfaces (together with the bond layer that also helps to fill gaps), reduce the extent of $\mathrm{S}_{0}$ mode generation; and ideally it also should be conductive to act as ground for the transducers, eliminating the need for additional electrodes. The use of a conductive membrane in combination with a conductive adhesive between the transducer elements and the membrane would be ideal, as it produces a repeatable and reliable ground with direct electrical connection to the transducers. If a nonconductive adhesive is used for the bonding between conductive membrane and elements, good signal strength depends critically on the control of the bondline thickness due to the capacitive coupling of the signal. The bond between membrane and structure is less critical, as it is not necessary or even advantageous to establish a conductive connection of the membrane (ground) to a metallic structure under investigation at the location of the array device.

Various types of materials (including standard rubber; silver-filled conductive rubber; latex; silver-filled, conductive latex; PVC; and PVDF) were tested in combination with different types of adhesives, mostly conductive and nonconductive epoxy and cyanoacrylates. The number of possible membrane and adhesive combinations was quickly reduced, as the requirements were good adhesion and transduction of the wave energy. For example, it proved to be almost impossible to get sufficient adhesion of the conductive, silver-filled, latex membrane to the specimen and transducer elements with the available adhesives. Another problem turned out to be the embrittlement of thin rubber membranes in combination with the cyanoacrylate adhesive. Cyanoacrylates and nonconductive epoxy were found to give a thin adhesive layer with good transduction properties. For further trials, it was decided to use a two-component, nonconductive epoxy setting at room temperature (Permabond, Somerset, NJ), as it was easier to use and gave repeatable results with good adhesion. For the bondline between the transducer and the membrane (ground), a small amount of conductive silver paint was added to the epoxy in order to achieve a conductive connection, while not altering the energy transduction properties. Nonconductive epoxy was used for the bondline between the membrane and structure; however, no effort was made to electrically separate the membrane (ground) from the structure.

The efficiency of the transducer, membrane, and adhesive combinations for the excitation of the $A_{0}$ mode was evaluated by the measurement of the out-of-plane displacement $400 \mathrm{~mm}$ away from the excitation transducer. Due to the different group velocities of the $\mathrm{A}_{0}$ and $\mathrm{S}_{0}$ guided wave modes, the pulses separate in time and their respective

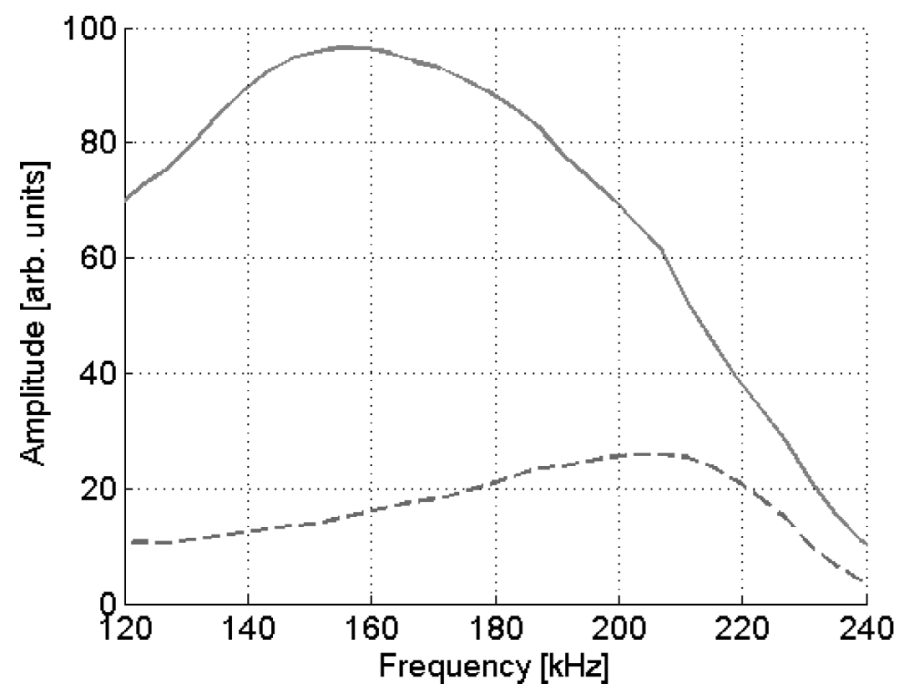

Fig. 1. Influence of coupling membrane on the amplitude of the excited $\mathrm{A}_{0}$ mode guided wave: direct bonding using two-component epoxy glue (solid line), $0.5 \mathrm{~mm}$ rubber membrane (dashed line); excitation transducer Pz27 disc (diameter $5 \mathrm{~mm}$, height $2 \mathrm{~mm}$ ), no backing.

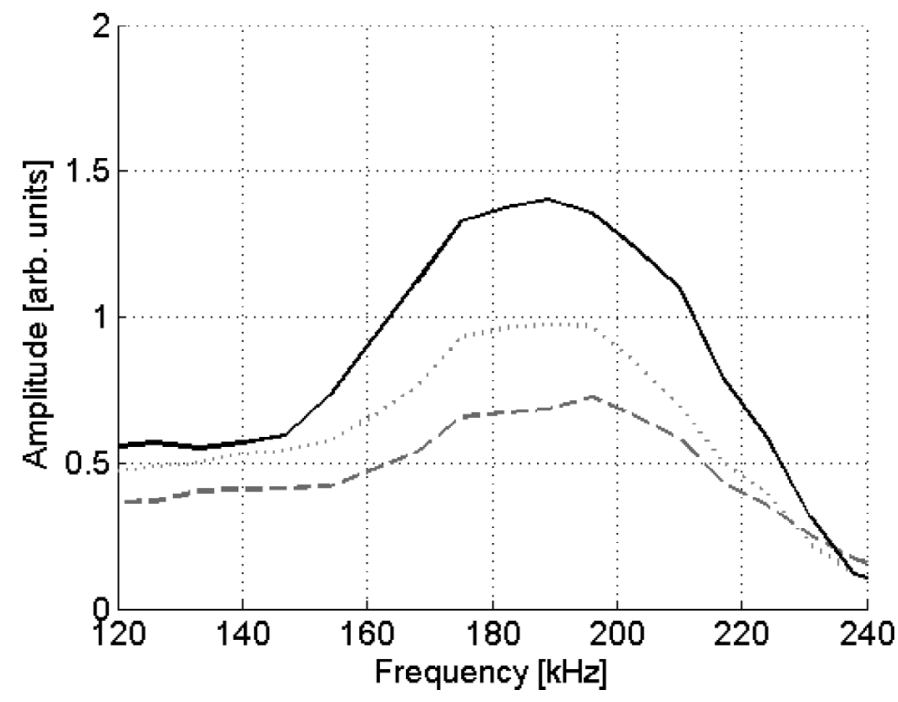

Fig. 2. Influence of thickness of brass backing mass on the amplitude of the excited $\mathrm{A}_{0}$ mode guided wave: length $6 \mathrm{~mm}$ (solid line), $4 \mathrm{~mm}$ (dotted line), $2 \mathrm{~mm}$ (dashed line); excitation transducer Pz27 disc (diameter $10 \mathrm{~mm}$, height $2 \mathrm{~mm}$ ) bonded to the plate via $0.16-\mathrm{mm}$ rubber membrane.

amplitudes can be calculated using a windowing function and fast Fourier transform (FFT). The measurement of the out-of-plane amplitude was performed using a heterodyne laser interferometer (Polytec, Waldbronn, Germany), which gives a repeatable transfer function and eliminates possible variations in the bonding of a measurement transducer. The excitation signal was a five-cycle toneburst with a center frequency varied in steps of $20 \mathrm{kHz}$ from $120 \mathrm{kHz}$ to $240 \mathrm{kHz}$, modulated by a Hanning window. The variation of the signal strength with frequency leads to slight jumps between the seven individual measurements evaluated in Figs. 1 and 2. The measurement procedure is 
described in more detail in [16]. Initial experiments on the bonding and design of the transducers were conducted on PZT discs polarized in the thickness direction of varying diameter $(5 \mathrm{~mm}, 7 \mathrm{~mm}$, and $10 \mathrm{~mm}$ ) and thickness $(0.5 \mathrm{~mm}, 1 \mathrm{~mm}$, and $2 \mathrm{~mm})$. Working well below the resonance frequency of the discs used (dependent on disc size), it was found that the comparison between performance with different types of adhesives and membrane and without membrane was little affected by disc size.

Fig. 1 shows the excited amplitude of the $\mathrm{A}_{0}$ mode guided wave for two coupling cases of a PZT disc polarized in the thickness direction (Pz27, diameter $5 \mathrm{~mm}$, thickness $2 \mathrm{~mm}$ ). The use of a $0.5-\mathrm{mm}$ rubber membrane clearly reduces the amplitude of the excited $\mathrm{A}_{0}$ mode by a factor of up to about 4 in the frequency range of interest, an unwanted effect; in pulse-echo mode this factor would square, and the signal to incoherent noise ratio would be lowered significantly. The maximum excitation voltage is limited by the electronics used and permitted power consumption. Within the linearity limits of the available electronics, it cannot be raised above about $100 \mathrm{~V}_{\mathrm{pp}}$ to achieve the desired toneburst. When working in frequency-thickness regime above $0.5 \mathrm{MHz}-\mathrm{mm}$ the $\mathrm{S}_{0}$ mode has a non-negligible, out-of-plane component and can be excited by the piezoelectric transducer. For the direct coupling of the disc to the plate, an out-of-plane amplitude of the $\mathrm{S}_{0}$ mode of about $10 \%$ of the $\mathrm{A}_{0}$ mode was measured between $120 \mathrm{kHz}$ and $240 \mathrm{kHz}$. The coupling membrane has the beneficial effect of reducing the amplitude of the $\mathrm{S}_{0}$ mode, which for the membrane shown here was not measurable below $180 \mathrm{kHz}$ and did not exceed $5 \%$ of the amplitude of the $\mathrm{A}_{0}$ mode in the frequency region of interest. Although most of the $\mathrm{S}_{0}$ mode signal is filtered out in the array algorithm (Section III) due to the larger wavelength, it still contributes to the coherent noise, and its excitation and reception should be minimized.

Several design variations, including the effect of a backing mass on improving the transducer performance, were evaluated. A significant increase in the amplitude of the excited guided wave is visible in Fig. 2, with a (radial) resonance effect around $180 \mathrm{kHz}$ with the $10-\mathrm{mm}$ diameter disc used in this particular test. The operating frequency is below the thickness resonance for all the backing thicknesses used, so the amplitude increases monotonically with backing thickness over the frequency range of interest. Due to the size/wavelength ratio restrictions detailed earlier, it was decided to work with PZT discs of diameter $5 \mathrm{~mm}$ and thickness $2 \mathrm{~mm}$. Testing the transducers with a brass backing mass of length $6 \mathrm{~mm}$ and various membranes, the best result (i.e., minimum amplitude reduction and good adhesion) was achieved in combination with a thin $(28 \mu \mathrm{m})$, silver-coated PVDF sheet, which also acts as ground connection for the transducers, avoiding the need for an additional ground electrode. The piezoelectric properties of the PVDF sheet were not exploited in this application, as both sides were connected to ground. The transducers were individually bonded to the PVDF sheet that covers the complete area under the array and acts as common ground. The transducers were held in place by holes in a Perspex (Lucite, Southampton, UK) plate with a slightly larger diameter $(5.1 \mathrm{~mm})$. This achieved a position accuracy of $0.1 \mathrm{~mm}$ or less than $1 \%$ of the wavelength used. The completed array with the PVDF sheet as a protective membrane then was bonded to the structure by applying adhesive only to the circular ring of the sheet directly underneath the transducers, but not to the center of the array (as adhesion of the membrane to the structure in that area was not necessary).

Initial trials showed reasonable repeatability of the measured transfer function with limited surface preparation and control of the bonding. The surface of the plate was polished with a fine sandpaper to remove corrosion and obtain a reasonably smooth surface. Just before bonding, all surfaces were cleaned using acetone and ethanol to remove dust and other residue. The two-component epoxy was applied by hand, and superfluous glue was removed by applying pressure on the transducer elements during curing. This resulted in a good reproducibility for individual test transducers, in which amplitude variations of about $10 \%$ were observed. However, the transfer function between individual transducer elements varied significantly more when they were assembled in the limited space of the array, with variations in amplitude of up to a factor 2 observed between the 32 transducer elements used. This is due to insufficient control of the bond layer thickness and pressure during the curing process and could be improved with appropriate jigging. It was observed that, by the application of a pressure to the individual transducers of about $1 \mathrm{MPa}$ by putting a large weight on the back of the array, these variations could be significantly reduced, and possible ringing of the transducers could be avoided. However, this may be impractical in a realistic setting. In the prototype, the electrical connections were made via the thin conductive PVDF membrane as common ground, and an individual spring (stiffness $1.17 \mathrm{~N} / \mathrm{mm}$ ) was connected to the backing mass of each transducer element. The spring mechanically decouples the individual transducer from the main array and helps to minimize mechanical cross talk between array elements.

\section{ArRay Algorithm AND Integration}

The chosen design of the guided wave array consists of a ring of 32 piezoelectric transducer elements, equally spaced on a diameter of $70 \mathrm{~mm}$ as shown in Fig. 3. This achieved a reasonably good angular resolution within the limitations for the manufacture of the transducer elements and multiplexing electronics. The circular array design was introduced to achieve the same performance in all directions. The developed array and multiplexing prototype shown in Fig. 3 was used, with external excitation and measurement instrumentation. The excitation signal was a 5 -cycle toneburst with a center frequency of $160 \mathrm{kHz}$ modulated by a Hanning window. Measurements also were performed for various excitation frequencies between $100 \mathrm{kHz}$ 

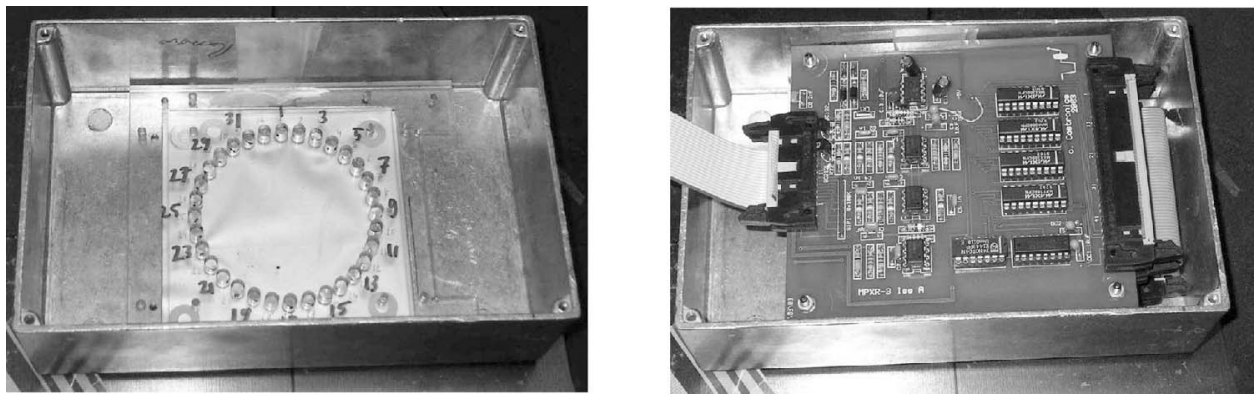

Fig. 3. Guided wave array prototype (left) and multiplexing electronics unit (right).

and $200 \mathrm{kHz}$ and showed good results, but no significant improvement of the defect detection sensitivity was observed. Multiplexing electronics were used to switch between the different excitation and receiving transducers. A time trace was measured and transferred to a computer for each combination of excitation and receiving transducer. Array operation and performance are discussed in more detail in [14].

The data from the array is first transformed to the frequency domain. The frequency domain data then is mapped to the wavenumber domain using the known dispersion relationship for the $A_{0}$ mode, to provide effective dispersion compensation [18]. A phased-addition algorithm is used to process the wavenumber domain data from the array. Effectively, the phased-addition algorithm synthesizes the wavenumber spectrum of a guided wave beam steered in any angular direction from the array. The circular symmetry of the array means that the imaging performance of the phased-addition algorithm, and hence its point spread function (PSF) is independent of angle. This in turn means that the data obtained from the phasedaddition algorithm can be deconvolved easily with its theoretical angular PSF, using angular Fourier transforms. This is described in more detail in [14]. The effect of the deconvolution is to suppress angular sidelobes. This is particularly important in the ring configuration of array elements used here, in which the sidelobes are unacceptably large when the phased-addition algorithm is used alone. The deconvolved data is Fourier transformed from the wavenumber domain to distance. The result is a radialangular B-scan, representing the information about the investigated structure in an easy-to-understand map.

The dynamic range of the monitoring device is limited physically by the performance of the transducer elements, and mathematically by the array layout and the reconstruction algorithm used. The number of elements used is limited by the number of channels in the multiplexing unit and the time and power consumption allowed for a single measurement. The maximum element spacing relative to the wavelength at the design frequency should not exceed half the wavelength, to avoid grating lobes. This limits the maximum diameter of the array for a given number of elements, and thus the angular performance of the array, as a larger array will have a better angular resolution and smaller side lobes [14].
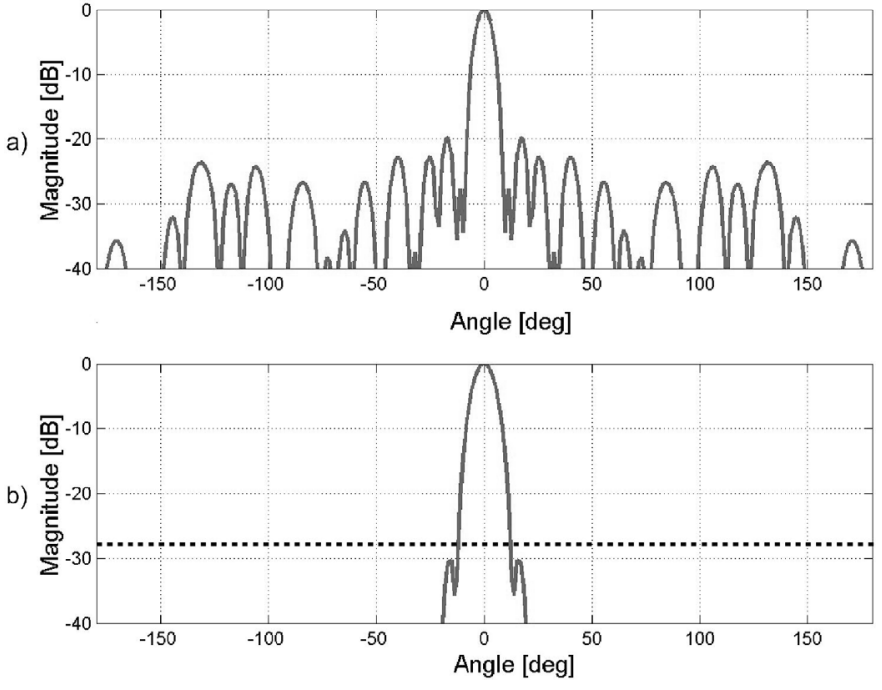

Fig. 4. Theoretically predicted angular performance of array design for a signal reflected back from the $0^{\circ}$ direction: (a) simple phasedaddition algorithm; (b) applying deconvolution algorithm. Dotted line shows experimentally achieved dynamic range of $-27 \mathrm{~dB}$.

For the available number of 32 elements and a maximum spacing of half the wavelength at the design frequency of $160 \mathrm{kHz}$, the theoretically achievable angular performance for a signal reflected back from the $0^{\circ}$ direction is shown in Fig. 4. In Fig. 4(a) the predicted dynamic range of about $20 \mathrm{~dB}$ for ideal transducer characteristics and applying only a simple phased-addition algorithm can be seen. The suppression of these sidelobes when the image is deconvolved is shown in Fig. 4(b). The main lobe drops to $-30 \mathrm{~dB}$ at $18^{\circ}$; beyond that the theoretical dynamic range of the array layout is better than $40 \mathrm{~dB}$, which would allow good defect resolution. The dynamic range actually achieved with the prototype was significantly smaller than theoretically predicted; only a dynamic range of $27 \mathrm{~dB}$ could be achieved. From the evaluation of the measured data, a significant amount of coherent noise and amplitude variations between the transducer elements of up to a factor of 2 were found.

\section{Laboratory Measurements}

Measurements were done on a 5 -mm thick steel plate (size: $2005 \mathrm{~mm}$ by $1020 \mathrm{~mm}$ ), shown in Fig. 5. The array 


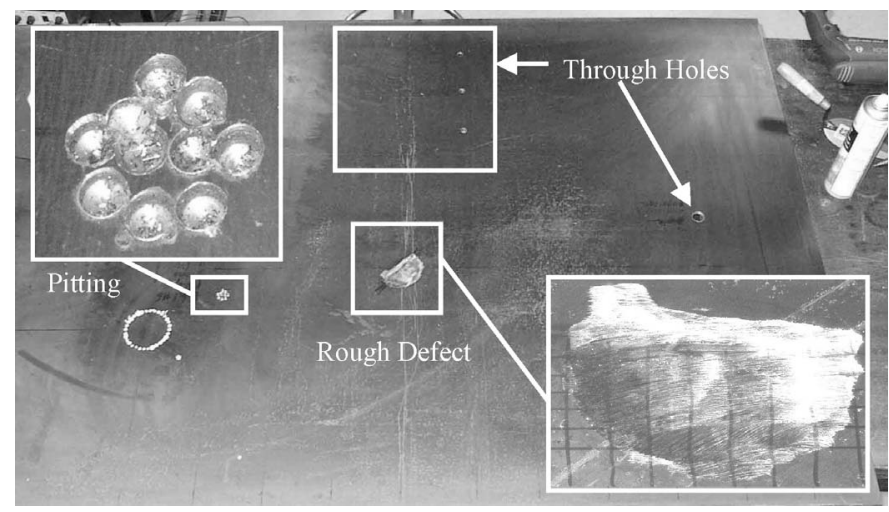

Fig. 5. Picture of the steel plate (thickness: $5 \mathrm{~mm}$; size: $2005 \mathrm{~mm}$ by $1020 \mathrm{~mm}$ ), with the location of the various defects marked; enlarged pictures of simulated corrosion pitting (10 part-through holes, $\oslash 5 \mathrm{~mm}, 25 \mathrm{~mm}$ by $20 \mathrm{~mm}$, depth $2-3 \mathrm{~mm}$ ) and simulated rough corrosion defect (machined using an angle grinder, $75 \mathrm{~mm}$ by $45 \mathrm{~mm}$, maximum depth $4 \mathrm{~mm}$ ).

a)

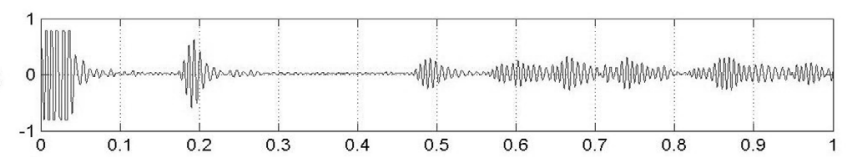

b)
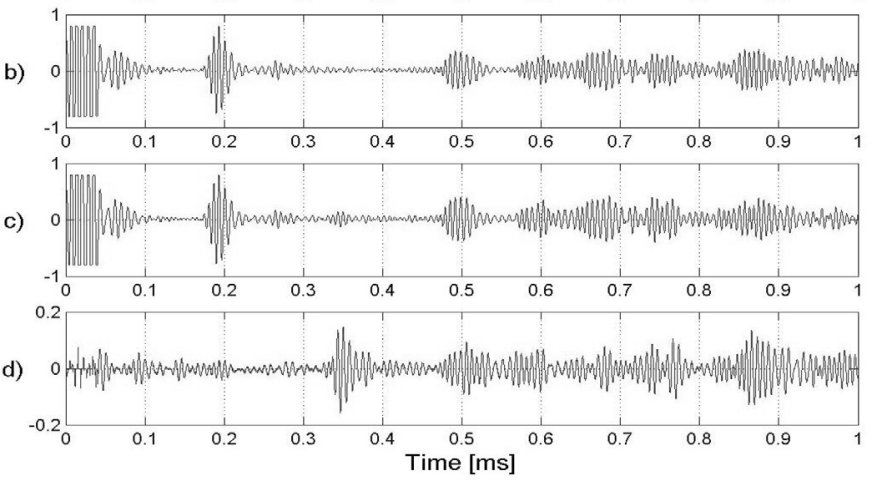

Fig. 6. Typical time signals sampled in pitch-catch mode using two transducers in the array; center frequency $160 \mathrm{kHz}$, five cycles, arbitrary amplitude scale: (a) no defect; (b) initial defects; (c) initial defects and grinded defect; (d) zoom of difference signal between (c) and (b).

device was bonded to the structure $300 \mathrm{~mm}$ from the long edge and $900 \mathrm{~mm}$ from the shorter edge. A variety of defects were machined into the plate to test the sensitivity and resolution of the monitoring approach. Five through holes [ $\oslash 5 \mathrm{~mm}, \oslash 10 \mathrm{~mm}$ (3 holes), and $\oslash 18.5 \mathrm{~mm}$ ] were drilled using a hand-held power drill. The type of rough defect produced by corrosion was simulated by using an angle grinder to produce a defect of varying depth $(75 \mathrm{~mm}$ by $45 \mathrm{~mm}$, maximum depth $4 \mathrm{~mm}$ ). Corrosion pitting was simulated as 10 part-through holes $(\oslash 5 \mathrm{~mm}$, depth 2 $3 \mathrm{~mm}, 25 \mathrm{~mm}$ by $20 \mathrm{~mm}$ ), as shown in Fig. 5 .

Measurements were taken after the machining of each defect to simulate long-term monitoring with various defects appearing in the structure. Typical time signals obtained are shown in Fig. 6. The initial time trace without defects in the structure is shown in Fig. 6(a). The reflec-

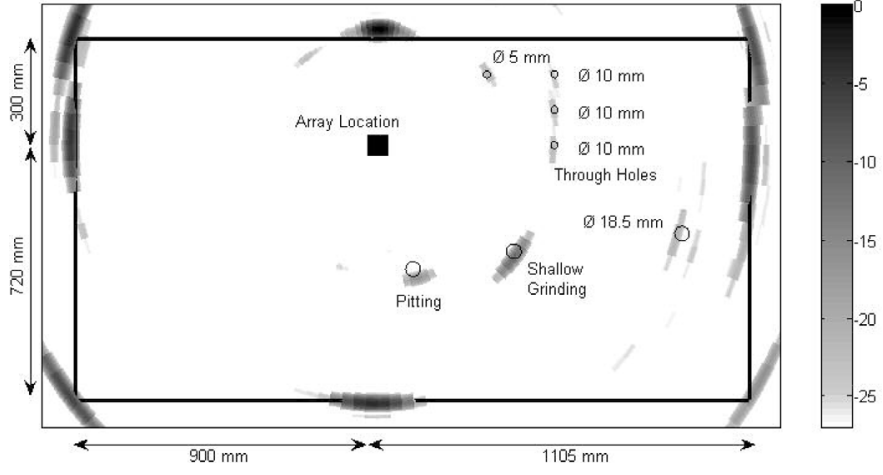

Fig. 7. Radial-angular B-scan of steel plate (edges marked) from indicated array position (distances in millimeters relative to array location), grayscale representing the correlation of signals reflected, center frequency $160 \mathrm{kHz}$; with defects and array positions marked, $27 \mathrm{~dB}$ scale.

tions from the two closest plate edges $(0.3 \mathrm{~m}$ and $0.72 \mathrm{~m}$ from array center) can be seen clearly at $0.19 \mathrm{~ms}$ and $0.49 \mathrm{~ms}$, with reflections from the various other plate edges and corners arriving later. After drilling several through holes into the plate, their reflections can be observed at $0.24 \mathrm{~ms}$ to $0.29 \mathrm{~ms}$ in Fig. 6(b). The additional signal due to the ground defect appears at $0.34 \mathrm{~ms}$ in Fig. 6(c). Taking the difference between the two signals shown in Fig. 6(b) and (c), respectively, the change in the received signal due to the ground defect can be observed more clearly in a zoom of the difference signal [Fig. 6(d)]. Such a measurement can be taken due to the monitoring nature of the observation, improving the signal-to-coherent noise ratio. However, from a single transducer combination, the defect cannot be localized exactly in the structure. Furthermore, the distinction between a true defect signal and other variations in the signal, as seen after $0.5 \mathrm{~ms}$ in Fig. 6(d) is very complicated, and defects located further away from the monitoring device might not be detected.

Applying the phased-array data processing described above, the results can be displayed as a radial-angular B-scan. The resulting B-scan for the plate with various defects is shown in Fig. 7, with the position of the array (square), the plate edges (lines), and defects (circles) marked. The amplitude is normalized to the maximum reflection (occurring at the closest plate edge) and shown on a grayscale down to $-27 \mathrm{~dB}$, the dynamic range of the array prototype. The measurement shows the reflections of the guided wave at the four sides and the four corners of the plate. The plate edges are seen only in the direction in which they are normal to the waves propagating radially from the array. The data processing algorithm is designed to pass signals transmitted and received along the same radial line and to reject signals from other directions. Clear, distinct reflections can be seen from the various defects, allowing their detection and localization in the structure. Good resolution between neighboring defects can be seen from the three distinct reflections of the three 10-mm diameter holes, located $100 \mathrm{~mm}$ apart. Defects that lie either close to the plate edges (5-mm diameter 
TABLE I

Measured and Predicted Amplitude of the Reflection of the Guided Wave at the Plate Edges in Decibel Scale, Normalized with the Maximum Reflection at the Closest Plate Edge.

\begin{tabular}{lccr}
\hline Location & Distance from array & Measurement & Prediction \\
\hline Upper plate edge (normalization) & $0.300 \mathrm{~m}$ & $0 \mathrm{~dB}$ & $0 \mathrm{~dB}$ \\
Lower plate edge & $0.720 \mathrm{~m}$ & $-2 \mathrm{~dB}$ & $-3.7 \mathrm{~dB}$ \\
Left plate edge & $0.900 \mathrm{~m}$ & $-5 \mathrm{~dB}$ & $-4.8 \mathrm{~dB}$ \\
Right plate edge & $1.105 \mathrm{~m}$ & $-6 \mathrm{~dB}$ & $-5.6 \mathrm{~dB}$ \\
\hline
\end{tabular}

hole, $100 \mathrm{~mm}$ from plate edge) or far from the measurement location (18.5-mm diameter hole, $0.922 \mathrm{~m}$ from the array) can be distinguished clearly, and thus detected in Fig. 7.

\section{Calibration Measurements and Comparison}

In order to ascertain the sensitivity of the array monitoring measurements, the predicted and measured reflection amplitude of the various structural features and defects is compared. Assuming that each transducer acts as a point source, from which the guided waves spread radially, the amplitude of the propagating guided wave is proportional to the reciprocal of the square root of distance from the source. Comparing the observed amplitudes of the reflections at the plate edges to the theoretical predictions in Table I, good agreement can be observed. The maximum difference occurs for the second closest plate edge, with a variation of $1.7 \mathrm{~dB}$. This error is not much larger than the estimated $1 \mathrm{~dB}$ accuracy of the amplitude of the measured reflections, and the accuracy of our predictions can be deemed to be in the order of $2 \mathrm{~dB}$.

The scattering of the first antisymmetric Lamb wave mode $\mathrm{A}_{0}$ at the circular through holes can be calculated analytically as described in [16], following a previous theoretical derivation [19]. The amplitude of the wave scattered back in the direction of the incident wave, measured using the array, depends on the ratios of wavelength, hole diameter, and plate thickness. For the case of constant plate thickness and wavelength (frequency) considered here, the amplitude of the back-scattered wave at the largest hole (diameter $18.5 \mathrm{~mm}$ ) is predicted to be $28 \%$ higher than for the 10-mm diameter holes and $70 \%$ higher than for the smallest hole (diameter $5 \mathrm{~mm}$ ). Allowing again for the geometric spreading of the guided wave due to the different distances of the holes from the measurement location (overall received amplitude proportional to the reciprocal of the distance), the measured amplitude of the backscattered guided wave can be predicted well, as shown in Table II. The measured amplitudes are displayed on the same decibel scale as Table I, normalized with the amplitude of the largest reflection at the plate edge. The differences between measured and predicted amplitude values are well within the accuracy limit of $2 \mathrm{~dB}$, and an indication of the severity of a defect can be obtained from the amplitude of the reflected signal, corrected for the propagation dis-
TABLE II

Measured and Predicted Amplitude of the Back-Scattered Guided Wave at the Circular Through Holes in Decibel Scale, Normalized with the Maximum Reflection at the Closest Plate Edge.

\begin{tabular}{rccc}
\hline $\begin{array}{c}\text { Hole } \\
\text { diameter }\end{array}$ & $\begin{array}{c}\text { Distance } \\
\text { from array }\end{array}$ & Measurement & Prediction \\
\hline $5 \mathrm{~mm}$ & $0.365 \mathrm{~m}$ & $-23 \mathrm{~dB}$ & $-22.7 \mathrm{~dB}$ \\
$10 \mathrm{~mm}$ & $0.500 \mathrm{~m}$ & $-24 \mathrm{~dB}$ & $-22.9 \mathrm{~dB}$ \\
$10 \mathrm{~mm}$ & $0.510 \mathrm{~m}$ & $-24 \mathrm{~dB}$ & $-23.1 \mathrm{~dB}$ \\
$10 \mathrm{~mm}$ & $0.538 \mathrm{~m}$ & $-25 \mathrm{~dB}$ & $-23.6 \mathrm{~dB}$ \\
$18.5 \mathrm{~mm}$ & $0.922 \mathrm{~m}$ & $-25 \mathrm{~dB}$ & $-26.1 \mathrm{~dB}$ \\
\hline
\end{tabular}

tance. The two simulated corrosion defects can be detected equally well. The simulated pitting was measured with a reflected amplitude of $-17 \mathrm{~dB}$ and the ground defect at $-13 \mathrm{~dB}$. This is well within the dynamic range of the array prototype of $-27 \mathrm{~dB}$, allowing for the reliable detection of such corrosion-like defects. No theoretical calculations for these defects were made, as the exact dimensions are difficult to measure and model, even in a finite-element model.

\section{Conclusions}

The prototype of a guided ultrasonic wave array for the structural integrity monitoring of large plate-like structures has been designed, built, and tested. The first antisymmetric Lamb wave mode $\mathrm{A}_{0}$ was excited and measured using piezoelectric transducer elements. The development of suitably small $A_{0}$ mode transducers and their reliable and repeatable bonding with a protective membrane to the structure under investigation has been described in detail.

The array design consists of a ring of 32 transducers permanently attached to the structure in a compact housing with the necessary multiplexing electronics. Using a phased-addition algorithm with dispersion compensation and deconvolution in the wavenumber domain, a good dynamic range can be achieved with a limited number of transducers. Due to limitations of the transducer performance, the achieved overall dynamic range was limited to $27 \mathrm{~dB}$, and further efforts in the transducer design and manufacturing will be necessary to improve the dynamic range for the detection of smaller defects.

Measurements were performed on a 5 -mm thick steel plate with a variety of machined defects to simulate cor- 
rosion damage. The measured reflections at model defects like circular through holes were comparable to theoretical predictions. Therefore, an approximate indication of the severity of a defect can be obtained from the amplitude of the reflected signal. Simulated corrosion pitting and a rough ground defect could be well detected within the dynamic range of the array measurement.

\section{REFERENCES}

[1] G. Benny, K. Steel, A. McNab, and G. Hayward, "A wireless sensor network of permanently installed structural integrity monitors," in AIP Conf. Proc. 760, 2005, pp. 1757-1764.

[2] D. E. Chimenti, "Guided waves in plates and their use in materials characterization," Appl. Mech. Rev., vol. 50, no. 5, pp. 247-284, 1997.

[3] P. Cawley, M. J. S. Lowe, D. N. Alleyne, B. Pavlakovic, and P. Wilcox, "Practical long range guided wave testing: Applications to pipes and rail," Mater. Eval., vol. 61, no. 1, pp. 66-74, 2003.

[4] D. N. Alleyne, B. Pavlakovic, M. J. S. Lowe, and P. Cawley, "Rapid long-range inspection of chemical plant pipework using guided waves," Insight, vol. 32, no. 2, pp. 93-96, 2001.

[5] I. A. Viktorov, Rayleigh and Lamb Waves: Physical Theory and Applications. New York: Plenum, 1967.

[6] X. L. Zhao and J. L. Rose, "Three-dimensional defect in a plate boundary element modeling for guided wave scattering," Adv. Nondestructive Eval., Key Eng. Mater., vol. 270-273, pp. 453460, 2004

[7] E. V. Malyarenko and M. K. Hinders, "Fan bean and double crosshole Lamb wave tomography for mapping flaws in aging aircraft structures," J. Acoust. Soc. Amer., vol. 108, no. 4, pp. 1631-1639, 2000.

[8] J. C. P. McKeon and M. K. Hinders, "Lamb wave scattering from a through hole," J. Sound Vib., vol. 224, no. 5, pp. 843862,1999

[9] O. Diligent, T. Grahn, A. Bostrom, P. Cawley, and M. J. S. Lowe, "The low-frequency reflection and scattering of the $\mathrm{S}_{0}$ Lamb wave mode from a circular through-thickness hole in a plate: Finite element, analytical and experimental studies," $J$. Acoust. Soc. Amer., vol. 112, no. 6, pp. 2589-2601, 2002.

[10] P. Wilcox, M. Lowe, and P. Cawley, "Omnidirectional guided wave inspection of large metallic plate structures using an EMAT array," IEEE Trans. Ultrason., Ferroelect., Freq. Contr., vol. 52, no. 4, pp. 653-665, 2005.

[11] K. Yamada and B. T. Khuri-Yakub, "Point contact ultrasonic transducer of waveguiding structure for high-frequency operation," Appl. Phys. Lett., vol. 68, no. 10, 1996.

[12] P. Fromme, P. D. Wilcox, M. J. S. Lowe, and P. Cawley, "A guided ultrasonic waves array for structural integrity monitoring," in AIP Conf. Proc. 700, 2004, pp. 142-149.

[13] P. Fromme and M. B. Sayir, "Detection of cracks at rivet holes using guided waves," Ultrasonics, vol. 40, no. 1-8, pp. 199-203, 2002.

[14] P. D. Wilcox, "Omni-directional guided wave transducer arrays for the rapid inspection of large areas of plate structures," IEEE Trans. Ultrason., Ferroelect., Freq. Contr., vol. 50, no. 6, pp. 699-709, 2003.

[15] B. D. Steinberg, Principles of Aperture and Array System Design. New York: Wiley, 1976.

[16] P. Fromme and M. B. Sayir, "Measurement of the scattering of a Lamb wave by a through hole in a plate," J. Acoust. Soc. Amer., vol. 111, no. 3, pp. 1165-1170, 2002.

[17] P. Fromme, P. D. Wilcox, and P. Cawley, "Remote monitoring of plate-like structures using guided wave arrays," in AIP Conf. Proc. 65\%. 2003, pp. 157-164.

[18] P. D. Wilcox, "A rapid signal processing technique to remove the effect of dispersion from guided wave signals," IEEE Trans. Ultrason., Ferroelect., Freq. Contr., vol. 50, no. 4, pp. 419-427, 2003.

[19] Y.-H. Pao and C. C. Chao, "Diffractions of flexural waves by a cavity in an elastic plate," AIAA J., vol. 2, no. 11, pp. 20042010, 1964.

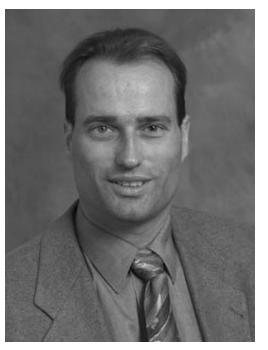

Paul Fromme was born in Schweinfurt, Germany, in 1971. He received a Dipl.-Ing. in mechanical engineering from the University of Karlsruhe, Karlsruhe, Germany, in 1996 and a Ph.D. degree (Dr.Sc. techn.) in mechanical engineering from the Swiss Federal Institute of Technology, Zurich, Switzerland, in 2001.

From 2002 to 2003, Dr. Fromme worked as a postdoctoral research associate in the nondestructive testing research group at Imperial College London, England, where he worked on the development of guided ultrasonic wave arrays for structural integrity monitoring.

Since 2003 he has worked as a lecturer in the Department of Mechanical Engineering at University College London, England.

His current research interests include guided ultrasonic wave propagation and scattering characteristics, fatigue defect monitoring, nondestructive testing applications, and biomedical applications of ultrasound.

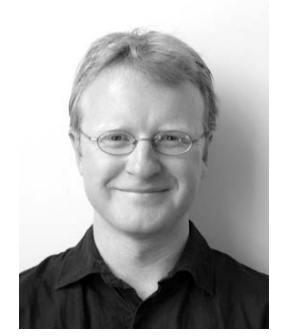

Paul D. Wilcox was born in Nottingham, England, in 1971. He received an M.Eng. degree in engineering science from the University of Oxford, Oxford, England, in 1994 and a Ph.D. degree from Imperial College, London, England, in 1998.

From 1998 to 2002 he was a research associate in the nondestructive testing research group at Imperial College where he worked on the development of guided wave array transducers for large area inspection. Since 2000 he also has acted as a consultant to Guided Ultrasonics Ltd., Nottingham, England, a manufacturer of guided wave test equipment.

Dr. Wilcox now works as a lecturer in dynamics at the University of Bristol, Bristol, England. His current research interests include long-range guided wave inspection, structural health monitoring, array transducers, and signal processing.

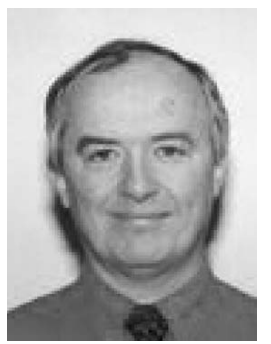

Michael J. S. Lowe was born in Belfast, UK, in 1957. He received a B.Sc. degree in civil engineering from the University of Edinburgh, Edinburgh, UK, in 1979, and M.Sc. and Ph.D. degrees in mechanical engineering from Imperial College, University of London, in 1987 and 1993, respectively. He has been a chartered member of the Institution of Mechanical Engineers since 1986. He is also a member of the British Institute of NonDestructive Testing, the Institute of Physics, and the French Society of Acoustics.

From 1979 to 1989 he worked in engineering consultancy, specializing in the application and development of numerical methods for the solution of problems in solid mechanics. His principal clients were in the nuclear power and offshore oil industries.

Since 1989 he has worked in the Department of Mechanical Engineering at Imperial College, University of London, and is currently a reader. His research interests are in the use of ultrasonic waves for non-destructive evaluation. His special areas of expertise are guided waves, the interaction of waves with defects and structural features, and numerical modeling. 


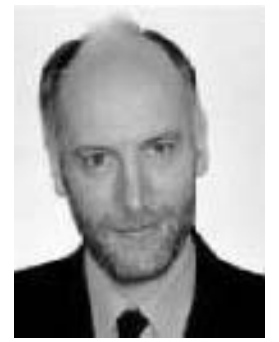

Peter Cawley (M'93) was born in Sheffield, England, in 1953. He received B.Sc. and Ph.D.

degrees in mechanical engineering from the University of Bristol, Bristol, England, in 1975 and 1979, respectively.

He worked in industry from 1979-1981, then joined the Department of Mechanical Engineering at Imperial College, London, initially as a lecturer, then successively senior lecturer, reader, and professor. He has worked on a wide variety of projects using sonic and ultrasonic methods applied to the inspection of adhesive joints, composite materials, mass produced components, aerospace structures, pipework, dental implants, and other structures. He has published over 120 refereed journal papers and a similar number of conference papers in this field and holds four current patents.

Dr. Cawley is a director of three spin-out companies set up to exploit technology developed in his research group (Guided Ultrasonics Ltd., Guided Ultrasonics (Rail) Ltd., and Integration Diagnostics Ltd.), and he is a consultant to a variety of industries. 